\title{
TELEREHABILITATION: POLICY ISSUES AND RESEARCH TOOLS
}

\author{
KATHERINE D. SEELMAN ${ }^{1} \&$ LINDA M. HARTMAN ${ }^{2}$ \\ ${ }^{1}$ DEPARTMENT OF REHABILITATION SCIENCE AND TECHNOLOGY, UNIVERSITY OF PITTSBURGH \\ ${ }^{2}$ HeAlth SCIEnCES Library System, University of PitTsburgh, PitTsburgh, PA
}

\section{ABSTRACT}

The importance of public policy as a complementary framework for telehealth, telemedicine, and by association telerehabilitation, has been recognized by a number of experts. The purpose of this paper is to review literature on telerehabilitation (TR) policy and research methodology issues in order to report on the current state of the science and make recommendations about future research needs. An extensive literature search was implemented using search terms grouped into main topics of telerehabilitation, policy, population of users, and policy specific issues such as cost and reimbursement. The availability of rigorous and valid evidence-based cost studies emerged as a major challenge to the field. Existing cost studies provided evidence that telehomecare may be a promising application area for TR. Cost studies also indicated that telepsychiatry is a promising telepractice area. The literature did not reference the International Classification on Functioning, Disability and Health (ICF). Rigorous and comprehensive TR assessment and evaluation tools for outcome studies are tantamount to generating confidence among providers, payers, clinicians and end users. In order to evaluate consumer satisfaction and participation, assessment criteria must include medical, functional and quality of life items such as assistive technology and environmental factors.

Keywords: Telerehabilitation, Telehomecare, Telepsychiatry, Telepractice

\section{INTRODUCTION}

The importance of public policy as a complementary framework for telehealth, telemedicine and, by association telerehabilitation, has been recognized by a number of experts (Weinstein et al, 2008, Wipf and Langner, 2006). Government health policy objectives involve quality, cost, and access to health service resources. Policy analysis and evaluation tools are used by government and the research community to determine the efficacy and efficiency of policies as a basis for decisions about resource allocations. The purpose of this paper is to review literature on telerehabilitation (TR) policy and research methodology issues in order to report on the current state of the science and to make recommendations about future research needs.

The population of interest for this review is people with disabilities across the age span. While few data systems identify them as a subpopulation, people with disabilities have been found to be in need of enhanced healthcare services. In Healthy People 2010 (U.S. Centers for Disease Control, n.d.), people with disabilities were described as a possible underserved population with larger-thanaverage health services utilization. Health disparities between people with and without disabilities have been identified, including excess weight, reduced physical activity, increased stress, and less frequent mammograms for women over age 55 years with disabilities. The percentage of the population that is disabled and has chronic disease increases sharply with age so that the numbers of persons needing health care assistance as a result of a disability is projected to increase dramatically (Cruise \& Lee, 2005). In turn, heightened demand for health services threatens to increase healthcare spending to unsustainable levels unless innovative strategies that curb costs are adopted. Therefore, the aging and disability demographic may be an incentive for the adoption of new and innovative health care delivery approaches such as telerehabilitation (TR) (Waters, 2005, Waters, 2008a, Waters, 2008b).

\section{BACKGROUND}

\section{DEFINITIONS}

This review uses the term telehealth as a generic designation for telehealth fields including telemedicine and telerehabilitation. Practitioners have long recognized the potential contributions that telehealth can make to 
equitable access to services, especially to rural and remote populations (Scalvini, Vitacca, Paletta, Giordano, $\&$ Balbi 2004). Telerehabilitation (TR) policy itself is largely, but not exclusively, embedded in telehealth and telemedicine policy. Evolving telepractice and the U.S. government agencies that administer telehealth, telemedicine, and telerehabilitation programs have developed operational definitions of these terms.

Telehealth is the use of electronic information and telecommunications technologies to support longdistance clinical healthcare, patient and professional health-related education, public health and health administration (U.S. Department of Health and Human Services Health Resources and Services Administration n.d). Telehealth delivery systems involve a complex set of elements that show considerable variability by technology, organizational models, clinical practice, and assessment and evaluation tools. Technologies used in telehealth typically are videoconferencing, the Internet, store-and-forward imaging, streaming media, and terrestrial and wireless communications (Center for Information Technology Leadership, 2007). Individuals with disabilities access health care using a variety of delivery systems ranging from acute medical/surgical hospital units to systems of care located in the home (Cruise \& Lee, 2005).

Telemedicine is the use of telecommunications technologies to help deliver healthcare services such as patient care and telemonitoring (Telemedicine Information Exchange, 2005). The practice of telemedicine involves devices that serve a myriad of clinical and patient objectives such as monitoring, diagnostics, communications and patient records. Applications include using computers to transmit data and images and using two-way interactive video so that isolated rural patients can receive medical advice from a doctor hundreds of miles away (U.S. Department of Health and Human Services Health Resources and Services Administration, 1994). Public policy does not have standard guidelines for coverage of devices that may include video cameras, phones, pagers and decision support tools (Waters, 2007).

The Rehabilitation Engineering Research Center on Telerehabilitation (2007) has defined TR as the remote delivery of rehabilitation and home health care services. TR services may include applications such as consultations, homecare, monitoring, therapy and direct patient care delivered to various locations including home, community, health facility and work settings (RERC on TR, 2007, Rosen, 2004, Winters, 1999). TR literature shows a broad appreciation of the utility of TR for serving populations that traditionally require physical rehabilitation, such as stroke, spinal cord injury, traumatic brain injury, and multiple sclerosis but also those that have communication, swallowing and hearing disorders and those who have psychiatric disorders (Baron, Hatfield \& Georgeadis, 2005, Theodorus and Russell,
2008, American Telemedicine Association Special Interest Group, 2006, Galea, Tumminia \& Garback, 2006, Baron, Brooke \& Georgeadis, 2005, Demiris, Shigaki \& Schopp, 2005 ; Egner, Phillips, Vora \& Wiggers, 2003, Kinsella, 1999, DeLisa, 1998 McCarthy \& Fox,2007 ). TR may optimize the timing, intensity and sequencing of intervention and provide the opportunity for the individual to continue to receive rehabilitation in their own social and vocational environment. While a literature review of policy and methodological publications will be implemented later, at this point it is appropriate to note that resolution of policy and methodological issues are made more challenging by their relationship to complex and often unresolved health care systems, technical and professional issues which often lie outside the public policy domain.

\section{SYSTEM COMPLEXITY AND TECHNICAL AND PROFESSIONAL ISSUES}

Telemedicine systems vary by population served, location, organizational model, technology, and evaluation frameworks, creating challenges for the design and conduct of cost and other studies that have real world reliability. Bashshur and colleagues (2005) observe that the telemedicine field is in a constant flux making it difficult to conceptualize and measure operating systems. Technological systems and configurations are dynamic and telemedicine organizational program models diverse, ranging from a single specialty service within a single delivery system, such as telerehabilitation, to a multi-specialty, multi-site network that offers a full range of medical and diagnostic services. Jennett and Andruchuk, (2001) identified key issues necessary to the successful integration of telehealth in the Canadian system as follows: (a) needs analysis, (b) business plan, (c) equipment, (d) evaluation, and (e) technical and professional policy standards. Theodorus and Russell (2008) note that some barriers and issues are generic to telehealth, such as professional portability and training. Other issues are more specific to the advancement of TR, such as degree of physical contact required in rehabilitation therapy, patient characteristics, and the availability of assessment and treatment tools that can replicate face-to-face practice. Assessment and treatment tools are used to collect data for outcome studies which are necessary to assess costs and benefits. In turn, evidence about costs and benefits is important to decisions about reimbursement. 


\section{HISTORY}

While the term telerehabilitation is relatively new, applications of telemedicine have a longer history going as far back as the 1880s when some physicians experimented with telecommunication technologies after the invention of the telephone in 1876 (Scalvini, et al, 2004). The U.S. government first supported telemedicine through services provided by agencies such as the U.S. Department of Veterans Affairs (VA), (U.S. Department of Veterans Affairs). The first recorded use of telemedicine in the VA was in 1957 for a telemental health project in Nebraska (Cooper et al, 2001). During the 20 years that ensued, other projects followed and flourished, leading the VA to begin its major systematic implementation of telemedicine in 1997. The VA began using the broader, more encompassing term telehealth in place of telemedicine in 2003. Telemedicine is a subset of VA telehealth, and VA telehealth is incorporated as one part of the wider rubric of VA care coordination. As of 2005, many of the Veteran's Health Administration's (VHA's) 21 Veteran's Integrated Service Networks (VISNs) were using some sort of TR technologies at VA medical centers and healthcare systems. These facilities use TR to augment services to community-based outpatient clinics (CBOCs) and Vet Center programs. They also connect to each other to provide both intra- and inter-VISN TRH referrals and specialty consultation so as to access rehabilitation care with direct services to veterans at home via videophones and remote health-monitoring devices (Department of Veterans Affairs).

In 1998, the National Institute on Disability and Rehabilitation Research (NIDRR) in the U.S. Department of Education funded the nation's first rehabilitation engineering research center on TR. NIDRR initiated research on TR as a complement to telemedicine and in response to a service delivery gap that emerged when managed-care policies truncated the length of inpatient rehabilitation stays. NIDRR also recognized the potential benefits of TR in areas of primary and secondary prevention for people with disabilities across the life span, health cost containment and vocational rehabilitation.

For the past 15 years, most non-veteran, federally funded telehealth programs have targeted rural populations, often with an emphasis on older adults (U.S. Department of Health and Human Services Health Resources and Services Administration). For example, the Office for the Advancement of Telehealth (OAT) in the Health Resources and Services Administration (HERSA) supports the Telehealth Network Grant Program to develop capacity for telehealth in rural areas for the medically underserved areas to improve and coordinate healthcare services. The Office of Rural Health Policy, HRSA, supports Rural Health Care Services Outreach Grant programs. The U.S. Department of Agriculture supports the Rural Development Distance Learning and Telemedicine (DLT) Loan and Grant Program (U.S. Department of Agriculture).

\section{METHODOLOGY}

As a preliminary step, telerehabilitation researchers at the Rehabilitation Engineering Research Center on Telerehabilitation were queried about policy issues that were important to their research and development projects. Websites of special interest groups on telerehabilitation, telemedicine, and telehealth at the American Telemedicine Association and the Rehabilitation Engineering and Assistive Technology Society of North America were examined to identify TR policy issues and research approaches. Using the results of these inquiries for guidance, a literature search strategy was developed and implemented. The searches resulted in close to 1,000 citations. The titles and abstracts were reviewed and approximately 325 abstracts were selected for further consideration. Of these, approximately 70 full articles were reviewed.

The search started with Ovid Medline (1950-2008 file) since it is the premier medical database and it has the controlled vocabulary MeSH (Medical Subject Headings). CINAHL (Cumulative Index to Nursing \& Allied Health Literature), covering 1982 to 2008, and PsychINFO, covering 1967-2008, were also searched using the Ovid interface.

The Advanced Ovid Search mode, along with the "Map Term to Subject Heading" feature, was used in each of the above databases. This provided the user with suggestions for possible subject headings when a non-subject heading term was entered. Additional search terms were found using the scope notes and the vocabulary trees for the controlled vocabulary. The terms were grouped into the main topics of telerehabilitation; policy; population of users; and a final one whose terms included the ideas of licensure, liability, and reimbursement. Known authors as well as known institutions make up the last two sets. These sets were then used in different combinations. When available the terms were searched as subject headings, otherwise they were searched as keywords using the .mp. command. Institutions were searched using the field code .in., for institution, as well as .mp.

The Scopus database contains citations from the scientific, technical, medical, and social sciences disciplines. The bulk of the references in Scopus are from 1996 to the present with some going back to the 1800s. A list of all the terms searched (e.g., subject headings, keywords, institutions) was created in MSWord. These can be found in the Appendix. Using the Advanced search box in Scopus the field code ALL was used for each term. In Scopus the ALL field command searches the abstract, affiliation, source title author, title, references, index terms and others. Again, the terms were placed into groups, such as policy and population. Each set was saved and used in various combinations. While Scopus gives results from reference lists, websites and patents, only those from the main result tab were 
examined. These included citations from journals, conferences, books and dissertations.

The University of Pittsburgh has the ability to do a federated search of databases in a particular discipline. The subjects of economics, government, international affairs, law and legal studies, sociology, and Western European studies were searched with the same terms used in the previous databases. Finally, a quick search was done in the Telemedicine Information Exchange (TIE) produced by the Association of Telehealth Service Providers. While it is only current up to 2006 , it provided a check on the legislative, law, and policy information already gathered.

Articles included for review had in the title or abstract actual mention or association with public policy and policy evaluation and research methods. Associational terms include the following: licensure; payment structures, particularly reimbursement; law, legislation or regulation. Those articles mentioning Medicare, Medicaid and other government programs or agencies were also selected from the retrieval. Initially the retrieval was limited to 1996-2008. In Scopus where the retrieval was rather large the dates were narrowed to 2001-2008 and review articles. Exclusion criteria included articles dealing with engineering and technology, medical and clinical interventions, adults under the age of 65 and children unless they had a disability; and articles not in English.

\section{RESULTS}

While there is a modest body of telerehabilitation literature on policy and policy methodology issues, there is a larger body of telehealth and telemedicine literature. Policy issues are often subsumed in sections within articles and other publications that address other topics. These articles routinely identified the following as important policy issues: cost, reimbursement, privacy and informed consent, fraud, liability, licensure, and systems security. With the exception of cost and reimbursement studies, the policy literature is largely descriptive and practical, often providing perspectives on strategies to generate a more supportive policy framework for telehealth, telemedicine, and telerehabilitation. Telehealth literature originates from locations around the world, particularly resource-rich countries, like the United States, Canada, Australia, Singapore and those located in Europe. It spans jurisdictional and interjurisdictional levels including global systems, regional, country and local or even multiple levels (Pak et al 2008, Scott, Chowdhury \& Varghese, 2002). In the United States, the Center for Telemedicine and e-Health Law (CTeL) (2005) and the American Telemedicine Association (2008) are among the organizations that have provided leadership in stimulating the generation of literature addressing research and practical perspectives on telehealth law, legislation, regulation and policy issues on the federal and state levels.

\section{LITERATURE ADDRESSING GENERAL POLICY ISSUES AND THEIR CONTEXT}

Theodorus and Russell (2008) provide an overview and perspective on the current state of telerehabilitation. They observe that while it may be possible to deliver rehabilitation services around the world, there are many key policy issues that must addressed. The issues, which are representative of those identified in other articles, are as follows: a) licensure and certification across state and national borders; b) equivalence of international clinical standards; c) regulation on privacy issues and the access and protection of patient health information; d) issues on costs and remuneration of services; e) liability and accountability; and f) unification of international rules effecting clinical consultations. Theodorus and Russell note that while a number of international organizations, such as the World Health Organization and the World Trade Organization, are entering the debate, there is a lack of leadership and focus on e-health policy.

Jennett, Scott, Affleck, Hailey, Ohinmaa, Anderson, C. et al. (2004) did a review of policy implications associated with the impact of telehealth on socioeconomic and health systems in Canada. They argue that telehealth cannot be viewed simply as an add-on service. It must be sensitive not only to cost and reimbursement issues but also consider issues such as social isolation, life stress, and poverty. Dena Puskin (2001, Puskin \& Urka 1999) a U.S. telehealth policy specialist observed that patients and clinicians living in rural and underserved areas will benefit from telehealth and telerehabilitation. However, adoption is slow because of policy and methodological barriers, such as standards and evaluation, and policy lags caused by technical standards and requirements of the Health Insurance Accountability and Portability Act (HIPAA). Also reaching back to 1999, Jack Winters elaborated on the now familiar litany of policy issues and barriers as follows: a) payment structures and reimbursement mechanisms do not support telehealth services; b) liability and whether or not the clinician, the provider or the telecommunications company is liable; c) quality standards for devices; and d) licensure and practice across U.S. state lines. In a review, Whitten and Sypher (2006) observed that the field has common barriers, especially the HIPPA because of the severe financial penalties for violations.

A number of articles use ethics and the socio technical aspects of telehealth as a "portal" to address policy issues. The content of these articles tend to be more philosophical. Authors are particularly sensitive to the vulnerability of disabled and older adults. These groups need accessible technology to access health information (Powell \& Lowe, 2005) and enhanced protection when serving as research subjects and as patients (Lehoux \& Blume, 2000, Marschollek, et al, 2007, Marziali, Dergal, \& McCleary , 2008). The homecare ethics literature reflects a general appreciation of the potential of remote 
delivery of homecare services to increase equitable distribution of health care resources while also expressing concern about the impact of a shift in service location on accountability and reportage, privacy, including family privacy, and the potential for a shift of workload from professionals located in health facilities to caregivers located at home. Authors also recommended the following: a) policy should inform clinical assessments of function and quality-of-life; and b) technical and clinical systems should be held accountable through reports about technology performance and the delivery of clinical services.

Kaplan and Litewka (2008), for example, identify the following policy-related problem areas: a) abridgement of privacy by inducing combining and mining data and implications of new technology on informed consent; b) inaccurate and obsolete data; c) security breaches; d) usability and user friendliness; e) data standards, and integration for linking patient and personal information to achieve interoperability of individual records, personal health management and public health; f) systems design and deployment decisions; and, g) tradeoffs between social isolation and enhanced care, especially homecare.

\section{LITERATURE ADDRESSING COST AND REIMBURSEMENT}

\section{COST}

Cost and reimbursement are among the most frequently named policy issues in the literature. Roine, Ohinmas \& Hailey ((2001) conducted a systematic review of telemedicine literature using economic assessment as one of their inclusion criteria. Of the 50 articles reviewed, few comprehensive economic analyses were identified and the quality of the analysis was described as relatively low. With few exceptions they reported that studies often lacked empirical background about the costs and benefits included in the studies. Because costs varied considerably among studies due to the diverse universe of technological and clinical options, the authors concluded that comparison of the cost estimates may not be feasible in many cases.

Cusack, Pan, Hook, Vincent, Kaelber, and Middleton (2008) at the Center for IT Leadership note that the lack of clarity about the value of telehealth has been one reason cited for slow adoption. The Center did a study of telehealth encounters in which there was a provider both with the patient and at a distance from the patient, using store-and-forward, real time video, and hybrid systems. Findings showed that the hybrid model was the most cost-effective and that its implementation in emergency rooms, prisons, nursing homes, and physician offices in the U.S. would save $\$ 4.3$ billion per year. Factors that most affected costs and savings were the cost of a faceto-face visit, the cost of a telehealth visit and the success rate of the telehealth visit, (i.e., the proportion of telehealth visits that avoided the need for face-to-face visits).

Another study (Rumberger \& Dansky, 2006) investigated the impact of telehealth on home health agencies (HHAs) in Pennsylvania. Findings indicate that telehealth can have a positive impact on HHA's financial position. In a randomized trial of telehealth interventions among people with mobility impairments resulting from spinal cord injury (Phillips, Vesmarovich, Hauber, Wiggers, \& Egner, 2001), patients received one of two telehealth interventions, or standard care. Findings indicate that telehealth interventions may be cost saving if program costs are more than offset by reduction in re-hospitalization. In a report on preliminary data from small telehealth initiatives, Vo (2008) at the AT\&T Center for Telehealth Research and Policy, reports promising results. The study uses a combination of "store-and-forward" technologies that transmit and interpret medical data with real-time video consultations linking the patient to one or more physicians such as the primary care physician and a remote specialist. This process eliminated the need for separate, follow up consultation with the patient and redundant laboratory tests ordered by multiple providers. Reduced consultations and numbers of lab tests ordered are regarded as significant sources of cost savings.

The Office for the Advancement of Telehealth (OAT) has funded a number of telehealth studies in areas such as mental health (Department of Health and Human Services, HERSA, 2006) and general patient health delivery (McCarthy \& Fox, 2007). The mental health study did not conduct rigorous outcome research but program evaluations suggest that telemental health programs improve continuity of care for rural consumers, increase family and consumer involvement in treatment, and reduce lengths of stays and re-admission rates in state psychiatric facilities. A case study at the University of Tennessee Health Science Center's Telehealth Network (McCarthy \& Fox, 2007), which received seed money from OAT, reported that it was able to transition projects to sustainability within the network because of demonstrated impact on access, quality and cost. Cost savings are shared. Patients have reductions in waiting time, time away from work and travel time. Third party payers reduce reimbursement for travel and provide more timely and appropriate treatment that averts costly complications. Providers' costs are reduced through efficiencies in the care process. The Network has received reimbursement for telehealth services by 35 third-party payers, including Medicaid, Blue Cross Blue Shield and commercial insurers at rates the same as, or in some cases higher than, in-person visits (McCarthy \& Fox, 2007) .

\section{REIMBURSEMENT}

The Center for Telemedicine and e-Health Law (CTeL) has a long history of looking at telehealth reimbursement. CTeL has published an independent sourcebook related to reimbursement for telemedicine. Its Reimbursement 
Sourcebook and State Telemedicine Reimbursement Guide address a broad range of legislative and regulatory issues involving telemedicine reimbursement policies through a variety of mechanisms at the federal and state levels, including, for example, Medicare, Medicaid, and private insurance -- all of which pay for telehealth in varying degrees. According to Robert Waters (2007) telemedicine reimbursement in the U.S. may be provided by the following: a) public payers, Medicare and Medicaid; b) private payers, fee for service; c) Managed Care, both public and private; and d) special payers such as government and worksite. Medicare reimbursementrelated issues include the following: a) expand eligible sites; b) expand eligible services; c) store and forward technology; d) expand geographic coverage; e) facility fee; e) co-payments; and f) home health DME (durable medical equipment), (Waters, 2007).

Hersh, Hickam, Servrance, Dana, Krages and Helfand, (2006) prepared a report on telemedicine and the Medicare population for the U.S. Agency for Healthcare Research and Quality. The goal was to inform decisions about coverage. Using a systematic but limited literature review of the efficacy of telemedicine services and usages for three categories of telemedicine services, the report concluded with the statement that "the promise of telemedicine is not matched by the strength of its evidence base" (p.36). Areas where telemedicine were found to be most promising include home health and specialties where care can be delivered via interactive videoconferencing such as psychiatry and neurology. Hersh et al. observed that the introduction of broadband connections to the home will have an impact on future research. The report asserts the need for evidence showing patient outcomes and any harm caused by telemedicine usage. However, it did acknowledge that there are instances when reimbursement or other incentives are not amenable to innovation, technical or otherwise.

In a study of private payer reimbursement for telemedicine services based on surveys of payers Whitten and Buis (2007) found expanded private reimbursement for telemedicine services, with 58 percent of responding organizations who provide potentially billable telemedicine services receiving private reimbursement. Whitten and Buis also found that 81 percent of those who receive private pay reported no differences between reimbursements for telemedicine services as compared to traditional face-to-face consultations. While this data shows increases in private coverage, the authors deemed it insufficient to generate widespread adoption of telemedicine services.

In a study of telehealth system use in nursing homes (Daly, Jogerst, Park, Kang \& Bae, 2005) the authors noted the lack of reimbursement for services from a nursing facility. The study also identified other restrictions including current procedural terminology (CPT) codes and location of the nursing facility. The CPT codes covered for telehealth services do not include the usual codes for nursing facility physicians and nurses. Kinsella (2008) observes that the Centers for Medicare and Medicaid Services (CMS) as well as most other health insurers require patients to be homebound which has a negative impact on home health services professions. Stein (2005) commented on a study of the use of virtual reality (VR) in telerehabilitation as a new treatment approach. He emphasized the importance of factors such as costeffectiveness to show whether or not VR can reduce the burden of disability and/or reduce reliance on high-cost one-on-one human therapy sessions. He added the caveat that clinicians need to make certain that the less tangible benefits of human-provided therapy including social support and companionship, are retained.

Gayle et al (2006) did a survey of state Medicaid health information technology reimbursement policies and a case study of Idaho's deliberations in setting telehealth and telemedicine reimbursement policy. Findings show that a lack of adequate provider reimbursement significantly slowed the growth of telemedicine in Idaho. In one of the more methodologically sophisticated policy studies retrieved for review, Schmeida, McNeal and Mossberger (2007) assessed the influence of traditional policy determinants on the extent of telehealth program implementation at the state level. The main dependent variable is the extent of telehealth program implementation at the state level across 29 medical specialty areas. Results suggest that state legislative professionalism, partisanship of state legislators, government resources, and severity of need are important factors. Nursing was found to be positively related to telehealth implementation and physician networks negatively associated. While procedural policy does not usually attract public interest, in this case, it did. Interest groups such as providers that must carry out the policy were mobilized. Palsbo (2004) did a study of current payment practice for telerehabilitation in state Medicaid programs using a telephone survey. Findings indicated that the primary reason for reimbursing for telemedicine is to make services available when there is no local practitioner. Seven states reimbursed telepsychology and four states reported reimbursing for telespeech and language pathology, physical therapy or occupational therapy. Consultation and evaluation and management services were most likely to be reimbursed. Study conclusions included the observation that TR is not enjoying widespread use, despite its potential benefit to people with disabilities who cannot travel to a clinic for rehabilitation. 


\section{LITERATURE ADDRESSING RESEARCH TOOLS AND METHODS}

Krupinski et al (2006) reported on the results of an expert panel convened at the request of the American Telemedicine Association to formulate a research agenda for the ATA. The request was based on the inconsistent quality of the existing body of literature which ranges from "purely anecdotal accounts of telehealth applications through well-controlled randomized clinical trials." The quality of the literature was thought to be contributing to slow adoption of telemedicine into the health care continuum. The article identifies four areas in which research is greatly needed, including the area of economic analysis. The authors commented on analytic tools, such as evaluation and research. They exclude telehealth program assessment and/or evaluation, a process applied to an individual project, because evaluations rarely can be generalized. Research is, of course, included because it refers to investigations that are generally hypotheses driven and generate results that can be generalized to the broader telehealth community. Research may include hypothesis driven quantitative studies and qualitative designs, such as Grounded Theory.

Applying these guidelines to economic analysis, the authors call for incorporating more robust analysis techniques into the field. They emphasize the following: a) the importance of framing the question and then creating a study design that parallels the question; and b) accounting for all cost elements. The authors recommend two priority areas phrased as follows: a) What are the short-and long-term impacts of telehealth on health care costs? and b) Creating better healthcare business and management models. In response to the question of the impacts of telehealth, the authors assert that analysis should flow from technology to ergonomics to clinical outcomes to economics. For example, once technology performance has been validated and ergonomically optimized, the clinical outcomes can determine whether patients are better maintained and avoid visits to the hospital. Economic analysis could then examine the long-term impacts of patient improvements on avoiding or delaying patient disability and the cost this has to society, Social Security and other programs.

Grigsby, Brega and Devore (2005) argue that Health Service Research (HSR) provides a valuable analytical framework for the assessment of telemedicine. The scope of HSR is broader than that of clinical trials and can focus on system of care, end-user acceptance, and outcomes, costs and access. The authors note that the field needs appropriate measures for each of these. They acknowledge that risk-adjustment is essential because of variables that may affect outcomes at different levels ("policy, system, technology, provider and patient risk factors" p. 327). Small sample size is a significant obstacle to analysis and interpretation of findings.
Grigsby, Brega and Devore acknowledge that randomized clinical trials may prove unfeasible but observe that appropriate quasi-experimental designs, such as casecontrol studies, can ascertain the possibility of specific outcomes. Using home healthcare as an example, they point to the availability of data from Centers for Medicare and Medicaid Services on utilization and outcomes.

Bashshur, Shannon and Sapci (2005) also point to the inadequacies of scientific rigor in evaluation studies. To address these problems, they posit two strategies which are not mutually exclusive to address these problems. The first strategy is to fund largescale experimental telemedicine programs and projects that can be designed to collect data sufficient to test specific dimensions and effects of the technology. This strategy assumes, among other factors, the availability of financial resources to support clinical trials. The second strategy is to use theoretical triangulation as one basis for assessing the impact of telemedicine so as to integrate results from both quantitative and qualitative research designs. The new strategy would combine established theory with cumulative data from research studies even though they may be based on imperfect designs. Hirsh, Hickam, Sevrance, Dana, Krages and Helfand (2006) argue that continued small or methodologically weak studies are unlikely to add to the evidence base for telemedicine. They recommended well-designed random clinical trials (RCTs), longitudinal observational studies and demonstration projects. Acknowledging the time and expense associated with RCTs, they recommend alternatives such as the use of electronic health records, wherein selective data could be extracted on patients with telemedicine interventions to assess them longitudinally.

Scheideman-Miller, Clark, Moorad, Post, Hodge \& Smeltzer (2002) reported on INTEGRIS, an Oklahomabased telemedicine program, initially supported by HERSA/OAT. The program has conducted a large randomized controlled study and small studies of groups of patients to assess effectiveness. The authors strongly support the need for a comprehensive telerehabilitation database of evidence on which to base studies to demonstrate the efficacy of telerehabilitation services to people with disabilities. They hope to create a nation-wide database looking at both clinical and efficacy criteria. 
CONCLUSIONS AND

\section{RECOMMENDATIONS FOR FUTURE RESEARCH}

This literature review provides a snapshot of the current state of the science in policy analysis for a fledgling telepractice area-telerehabilitation-as well as telehealth. The literature search did not identify a significant body of TR or even telehealth policy-related literature. The policy literature identified was routinely descriptive. Policy was most often relegated to a summary section on barriers to adoption late in a research-related publication committed to a different subject. Within the literature the following policy issues were most frequently named: costs, reimbursement, licensure, privacy, security, liability and fraud. In the areas of traditional policy research such as legislative behavior, decision-making and interest groups, the literature provided some evidence to support the need for further study because professional groups and legislators can create formidable barriers to adoption of telehealth.

The field of telehealth, including TR, must meet the challenge of more clearly delineating cost items, irrespective of the scope of the study. Medicare and Medicaid and other public programs that make decisions about service reimbursement require cost calculations based on evidence generated by rigorous outcome and efficacy studies that show improvements in function and reduced utilization of health care services. Among the various telehealth applications areas, home care may be particularly promising for TR research. While the review did not identify studies in rehabilitation counseling, they, too, should be pursued based on the promising evidence from studies of telepsychiatry and mental health.

Even with the burgeoning older adult demographic, rehabilitation research continues to be vastly underfunded. Rehabilitation research has routinely suffered from small sample size, in part due to inadequate research support for large-scale studies. Strategies to compensate for traditional research inadequacies can adopt triangulation of quantitative and qualitative research and cross-institutional collaboration. TR case studies should more rigorously explore the effectiveness and efficacy of delivering services at home to traditional rehabilitation populations, including those with chronic illness using large databases such as Medicare and state Medicaid patient records, when available. The literature did not reference the International Classification on Functioning, Disability and Health (ICF). In order to evaluate consumer satisfaction and participation, assessments criteria must include medical, functional and quality of life items such as assistive technology and environmental factors.

As a telepractice area operating worldwide and responding to ever increasing demands for delivery of expert consultations and other services, TR must be knowledgeable about and involved with general telehealth policy issues as well as issues peculiar to rehabilitation. Researchers and practitioners are familiar with the traditional degree of physical contact required in physical rehabilitation therapy, patient characteristics, and the availability of assessment and treatment tools used in face-to-practice. Now protocols are required and studies generated that compare face-to-face practice outcomes with those generated in remote delivery of services. These challenges are far exceeded by the potential to realize a vision of a future with more available, accessible and affordable high-quality health, especially for underserved populations, with the full continuum of care.

Acknowledgement: We want to acknowledge the early assistance provided by Danny Fernandez, a Ph.D. student at Carnegie Mellon University.

\section{CONTRIBUTIONS FROM THE STATE OF THE SCIENCE ON TELEREHABILITATION: PUBLIC POLICY ISSUES AND RESEARCH TOOLS}

Eighty-two people (not including panelists and staff) from seven countries (Australia, Canada, Philippines, Spain, The Netherlands, UK, USA) and 26 states in the U.S. participated on the final, fourth day of the State of the Science Conference on Telerehabilitation. Participants identified themselves within a broad range of professions including health (physicians, nurses), rehabilitation (physical therapists, occupational therapists, rehabilitation engineers, speech-language pathologists, and vocational rehabilitation counselors), health information, business and industry (assistive technology), pharmacy, and academia (research/scientist, faculty, students).

Audience dialogue and recommendations are captured in the following summary of comments. A lively dialogue developed around cost. One commenter observed that cost effectiveness could not be determined without more information about reimbursement but at least in the area of seating and mobility, we do not have a clear picture of reimbursement. Transportation costs, now absorbed by clients and patients in face-to face service delivery, should not be considered a cost-saving factor in remote health service delivery because insurers are not paying for it now. However, Worker's Compensation and Medicaid programs that pay for tax services have some financial incentives for tele-care. While most participants seemed to agree that telerehabilitation would improve access to rehabilitation, they also indicated that the potential for increasing demand made most insurers afraid of telemedicine/telerehabilitation. Insurers cannot quantify unmet needs and they are also afraid of the potential for fraud and abuse, especially with store-and-forward practice patterns. One participant recommended a literature review of tele-care coordination and another 
asked about the position of the AMA on telemedicine.

The telerehabilitation policy panel was very well received with narrative responses indicating an appreciation for the level of expertise and the diversity of perspectives. Audience participants were strongly favorable in response to a question about whether or not they would recommend this session to others. They also indicated increased understanding of the following: a) need for policy as a complement to technology and clinical services; b) need for outcome studies; c) need for innovation in health care to meet the needs of the world's burgeoning older adult and disability populations; d) need for including medical, functional and quality of life factors into studies; and f) need for a data base of research studies and research tools.

\section{ACKNOWLEDGEMENT}

We want to acknowledge the early assistance provided by Danny Fernandez, a Ph.D. student at Carnegie Mellon University.

\section{REFERENCES}

American Telemedicine Association. (2008). ATA American Telemedicine Association. Retrieved October 1, 2008, from http://www.atmeda.org/

American Telemedicine Association Special Interest Group. (2006). Telerehabilitation Bibliography, Spring 2006. Retrieved October 11, 2008, from http://www. atmeda.org/icot/sigtelerehab.htm

Baron, C., Hatfield, B., \& Georgeadis, A. (2005). Management of communication disorders using family member input, group treatment, and telerehabilitation. Top Stroke Rehabil, 12(2), 49-56.

Bashshur, R., Shannon, G., \& Sapci, H. (2005). Telemedicine evaluation. Telemed J E Health, 11(3), 296316.

Center for Information Technology Leadership. (2007). The value of provider-to-provider telehealth technologies. Retrieved October 20, 2008, from http://www.atmeda. org/news/ValueofProvidertoProviderTech.pdf

Center for Telemedicine and e-Health Law. (2005). Telehealth \& Emerging Technologies - Reimbursement. Retrieved October 13, 2008, from http://www.ctel.org/ Reimbursement.html

Cooper, R. A., Fitzgerald, S. G., Bonninger, M. L., Brienza, D. M., Shapcott, N., Cooper, R., et al. (2001). Telerehabilitation: Expanding access to rehabilitation expertise. Proceedings of the IEEE, 89(8), 1176-1191.

Cruise, C. M., \& Lee, M. H. M. (2005). Delivery of rehabilitation services to people aging with a disability. Physical Medicine and Rehabilitation Clinics of North America, 16(1), 267-284.

Cusack, C. M., Pan, E., Hook, J. M., Vincent, A., Kaelber, D. C., \& Middleton, B. (2008). The value proposition in the widespread use of telehealth. J Telemed Telecare, 14(4), 167-168.

Daly, J. M., Jogerst, G., Park, J. Y., Kang, Y. D., \& Bae, T. (2005). A nursing home telehealth system: keeping residents connected. J Gerontol Nurs, 31(8), 46-51.

DeLisa, J. A. (Ed.). (1998). Rehabilitation Medicine: Principles and practice (3rd ed.). New York: LippincottRaven.

Demiris, G., Shigaki, C. L., \& Schopp, L. H. (2005). An evaluation framework for a rural home-based telerehabilitation network. J Med Syst, 29(6), 595-603.

Egner, A., Phillips, V. L., Vora, R., \& Wiggers, E. (2003). Depression, fatigue, and health-related quality of life among people with advanced multiple sclerosis: results from an exploratory telerehabilitation study. NeuroRehabilitation, 18(2), 125-133.

Galea, M., Tumminia, J., \& Garback, L. M. (2006). Telerehabilitation in spinal cord injury persons: A novel approach. Telemedicine Journal \& E-Health, 12(2), 160162.

Gray, G. A., Stamm, B. H., Toevs, S., Reischl, U., \& Yarrington, D. (2006). Study of participating and nonparticipating states' telemedicine Medicaid reimbursement status: Its impact on Idaho's policymaking process. Telemed J E Health, 12(6), 681690.

Grigsby, J., Brega, A. G., \& Devore, P. A. (2005). The 
evaluation of telemedicine and health services research. Telemed J E Health, 11(3), 317-328.

Hersh, W. R., Hickam, D. H., Severance, S. M., Dana, T. L., Krages, K. P., \& Helfand, M. (2006). Telemedicine for the Medicare population: Update. Evid Rep Technol Assess (Full Rep)(131), 1-41.

Jennett, P. A., \& Andruchuk, K. (2001). Telehealth: 'real life' implementation issues. Comput Methods Programs Biomed, 64(3), 169-174.

Jennett, P. A., Scott, R. E., Affleck Hall, L., Hailey, D., Ohinmaa, A., Anderson, C., et al. (2004). Policy implications associated with the socioeconomic and health system impact of telehealth: A case study from Canada. Telemed J E Health, 10(1), 77-83.

Kaplan, B., \& Litewka, S. (2008). Ethical challenges of telemedicine and telehealth. Cambridge Quarterly of Healthcare Ethics, 17(4), 401-416.

Kinsella, A. (1999). Disabled populations \& telerehabilitation--new approaches. Caring, 18(8), 2022.

Kinsella, A. (2008). The Home Telehealth Primer. Retrieved October 10, 2008, from http://tie.telemed.org/articles/ article.asp?path=articles\&article=homeTelehealthPrim er_ak_tie08.xml

Krupinski, E., Dimmick, S., Grigsby, J., Mogel, G., Puskin, D., Speedie, S., et al. (2006). Research recommendations for the American Telemedicine Association. Telemedicine Journal \& E-Health, 12(5), 579-589.

Lehoux, P., \& Blume, S. (2000). Technology assessment and the sociopolitics of health technologies. Journal of Health Politics, Policy \& Law, 25(6), 1083-1120.

Marschollek, M., Mix, S., Wolf, K. H., Effertz, B., Haux, R., \& Steinhagen-Thiessen, E. (2007). ICT-based health information services for elderly people: Past experiences, current trends, and future strategies. Medical Informatics and the Internet in Medicine, 32(4), 251-261.

Marziali, E., Serafini, J. M. D., \& McCleary, L. (2005). A systematic review of practice standards and research ethics in technology-based home health care intervention programs for older adults. Journal of Aging \& Health, 17(6), 679-696.

McCarthy, D., \& Fox, K. (2007). Case study: University of Tennessee Health Science Center's Telehealth Networks. Retrieved October 10, 2008, from http:// www.commonwealthfund.org/innovations/innovations_ show.htm?doc_id=468916

Pak, H. S., Brown-Connolly, N. E., Bloch, C., Clarke, M., Clyburn, C., Doarn, C. R., et al. (2008). Global forum on telemedicine: Connecting the world through partnerships. Telemedicine Journal \& E-Health, 14(4), 389-395.

Palsbo, S. E. (2004). Medicaid payment for telerehabilitation. Arch Phys Med Rehabil, 85(7), 11881191.

Phillips, V. L., Vesmarovich, S., Hauber, R., Wiggers, E., \& Egner, A. (2001). Telehealth: reaching out to newly injured spinal cord patients. Public Health Rep, 116 Suppl 1, 94-102.

Powell, J. A., Lowe, P., Griffiths, F. E., \& Thorogood, M.
(2005). A critical analysis of the literature on the Internet and consumer health information. J Telemed Telecare, 11 Suppl 1, 41-43.

Puskin, D. S. (2001). HHS Perspective on Telehealth. Retrieved October 5, 2008, from http://www.ieeeusa. org/committees/MTPC/Saint2001/saint2001puskin.ppt

Puskin, D. S., \& Urka, M. A. (1999). A policy maker's glimpse of the road ahead: Telerehabilitation in the $21 \mathrm{st}$ century. Top Spinal Cord Inj Rehabil, 5(3), 70-74.

Rehabilitation Engineering Research Center on Telerehabilitation. (2007). Telerehabilitation: meeting consumer needs through rehabilitation engineering research. Retrieved October 18, 2008, from http://www. atmeda.org/news/2006_presentations/t4c3.ppt

Roine, R., Ohinmaa, A., \& Hailey, D. (2001). Assessing telemedicine: A systematic review of the literature. Cmaj, 165(6), 765-771.

Rosen, M. J. (2004). Telerehabilitation. Telemed J E Health, 10(2), 115-117.

Rumberger, J. S., \& Dansky, K. (2006). Is there a business case for telehealth in home health agencies? Telemed J E Health, 12(2), 122-127.

Scalvini, S., Vitacca, M., Paletta, L., Giordano, A., \& Balbi, B. (2004). Telemedicine: A new frontier for effective healthcare services. Monaldi Arch Chest Dis, 61(4), 226233.

Scheideman-Miller, C., Clark, P. C., Moorad, A., Post, M. L., Hodge, B. G., \& Smeltzer, S. (2002). Efficacy and sustainability of a telerehabilitation program. Retrieved October 5, 2008, from http://portal.acm.org/citation.cfm ?id=820753.821657\& $\mathrm{jmp}=\mathrm{cit} \& \mathrm{col}=\mathrm{GUIDE} \& d \mathrm{~d}=\mathrm{GUIDE} \& \mathrm{C}$ $\mathrm{FID}=8595260 \&$ CFTOKEN $=80249238 \# \mathrm{CIT}$

Schmeida, M., McNeal, R., \& Mossberger, K. (2007). Policy determinants affect telehealth implementation. Telemed J E Health, 13(2), 100-107.

Scott, R. E., Chowdhury, M. F., \& Varghese, S. (2002). Telehealth policy: looking for global complementarity. J Telemed Telecare, 8 Suppl 3, S3:55-57.

Stein, J. (2005). Commentary: J. Stein. CyberPsychology \& Behavior, 8(3), 216-217.

Telemedicine Information Exchange. (2005). Telemedicine 101. Retrieved October 10, 2008, from http://tie.telemed. org/articles/article.asp?path=telemed101\&article=tmco ming_nb_tie96.xml

Theodorus, D., \& Russell, T. (2008). Telerehabilitation: Current perspectives. In R. Latifi (Ed.), Current principles and practices of telemedicine and e-Health (pp. 119-209). Amsterdam: IOS Press.

U.S. Centers for Disease Control. (n.d.). Healthy People 2010. Chapter 6. Disability and secondary conditions.

U.S. Department of Health and Human Services Health Resources and Services Administration. (1994). Rural Telemedicine Projects. Retrieved September 30, 2008, from http://www.dhhs.gov/news/press/ pre1995pres/941110a.txt

U.S. Department of Health and Human Services Health Resources and Services Administration. (n.d.). Telehealth. Retrieved September 18, 2008, from http:// www.hrsa.gov/telehealth/

U.S. Department of Health and Human Services Health Resources and Services Administration. (2006). 
TeleHealth for Kansans (OAT Grant). Retrieved October 10, 2008, from http://www2.kumc.edu/ telemedicine/2008Programs/telehealthforkansans.htm

U.S. Department of Veterans Affairs. (2008). Office of Care Coordination Services Home Telehealth. Retrieved September 30, 2008, from http://www.carecoordination. va.gov/

Vo, A. H. (2008). The telehealth promise: Better health care and cost savings for the 21st century. Retrieved October 9, 2008, from http://ehealthvirginia.org/ downloads/20080529/UTMB\%20Telemedicine\%20 White\%20Paper\%2019May2008.pdf

Waters, R. J. (2007). Let's make a deal -- How to structure telehealth ventures. Retrieved October 8, 2008, from http://www.ctel.org/CTeLsSeminaratATA.html

Waters, R. J. (2008). Telehomecare is essential to longterm home care. Caring: National Association for Home Care magazine, 27(2), 52-53.

Waters, R. J. (2008). A tripartite relationship for home care in the future. Caring : National Association for Home Care magazine, 27(1), 48-49.

Waters, R. J., \& Eder-Van Hook, J. (2005). Retiring baby boomers create opportunities for innovation. Caring : National Association for Home Care magazine., 24(10), 84-86.

Weinstein, R. S., Lopeez, A. M., Krupinski, E. A., Beinar, S. J., Holcomb, M., McNeely, R. A., et al. (2008). Integrating telemedicine and telehealth: Putting it all together. In Current principles and practices of telemedicine and e-Health (pp. 23-38). Amsterdam: IOS Press.

Whitten, P., \& Buis, L. (2007). Private payer reimbursement for telemedicine services in the United States. Telemed J E Health, 13(1), 15-23.

Whitten, P., \& Sypher, B. D. (2006). Evolution of telemedicine from an applied communication perspective in the United States. Telemed J E Health, 12(5), 590-600.

Winters, J. (1999). Workshop on home care technologies for the 21st century. Retrieved October 15, 2008, from www.eng.mu.edu/wintersj/jack/pubs.htm

Wipf, K. R., \& Langner, B. (2006). Policy approaches to chronic disease management. Home health care management practice, $18(452-462)$.

\section{APPENDIX}

The terms for each section were ORed together and the resulting set was used in the combinations with the other sets.

\section{TELE TERMS}

Telerehabilitation

Rehabilitation AND telemedicine

Rehabilitation AND remote consultation

Telemedicine

Telehealth

Telecommunications AND home care services

Home care services AND remote consultation

Telemedicine AND home care services

Telepractice

Telemonitoring

Teletherapy

Teleservices

Telemetry

Telecommunications AND (public health practice OR delivery of health care OR health practice)

Telecommunications AND clinical care

Telecommunications AND health care

Telecommunications AND healthcare

POLICY TERMS

Public policy

Health policy

Social policy

Policy

Resource allocation

Health care rationing

Material allocation

Service allocation

Policy evaluation

Healthcare policy

Health care policy

POPULATION TERMS

People with disabilities

Persons with disabilities

Person with disabilities

(disabled persons OR amputees OR disabled children OR

hearing impaired persons OR mentally disabled persons OR

mentally ill persons OR visually impaired persons)

Physically challenged

Physically handicapped

Physically disabled

Handicapped

Aged

Older adults

"Aged, 80 and over"

Elderly

(stroke OR brain infarction OR brain stem infarctions OR lateral medullary syndrome OR cerebral infarction OR dementia, anterior cerebral artery OR infarction, middle cerebral artery OR infarction, posterior cerebral artery) (brain injuries OR brain concussion OR post-concussion syndrome OR brain hemorrhage, traumatic OR brain stem hemorrhage, traumatic OR cerebral hemorrhage, traumatic OR brain injury, chronic OR diffuse axonal injury OR epilepsy, post traumatic OR pneumocephalus)

(Spinal cord injuries OR central cord syndrome) 
(Traumatic brain injury OR traumatic brain injuries)

(spinal cord injury OR spinal cord injuries)

\section{Licensure, Health Care Practice, etC.}

TERMS

(licensure OR licensure, hospital OR licensure, medical OR

licensure, nursing)

(credentialing OR accreditation OR "joint commission on accreditation of healthcare organizations OR certification) ("quality of health care" OR guideline adherence OR program evaluation OR quality assurance, health care OR benchmarking OR clinical audit OR medical audit OR guidelines as topic OR "codes of ethics" OR practice guidelines as topic OR quality indicators, health care) (insurance, health, reimbursement OR reimbursement mechanisms)

Privacy

Personal space

Confidentiality

(computer security OR system security)

(liability, legal OR insurance, liability)

Interoperability

Ethics

\section{AUTHORS}

(Speedie sm OR Stuart M Speedie OR speedie s OR stuart speedie)

(Doolittle gc OR gary c doolittle OR Doolittle g OR gary

doolittle)

(Winters jm OR jack m winters OR winters j OR jack winters)

(Rosen m OR Michael rosen)

INSTITUTIONAL TERMS

"department of agriculture"

Rural development distance learning

"office for the advancement of telehealth"

("health resources and services administration" OR HRSA)

Telehealth network grant program

"office of rural health policy"

Rural health care services outreach

"national academy of sciences"

("centers for medicare Medicaid services" OR "centers for medicare Medicaid services" OR cms hhs)

("agency for healthcare research and quality" OR ahrq)

"rehabilitation engineering research center on

telerehabilitation"

American telemedicine association

(resna OR "rehabilitation engineering and assistive

technology society")

Veterans administration medical center

Veterans administration

"department of veterans affairs" 